\title{
Cardiovascular Outcomes in Action to Control Cardiovascular Risk in Diabetes: Impact of Blood Pressure Level and Presence of Kidney Disease
}

\author{
Vasilios Papademetriou ${ }^{a}$ Misbah Zaheer $^{b}$ Michael Doumas $^{c}$ Laura Lovato $^{d}$ \\ William B. Applegate ${ }^{e}$ Costas Tsioufis $^{a}$ Amy Mottle $^{f}$ Zubin Punthakee ${ }^{h}$ \\ William C. Cushman ${ }^{9}$ ACCORD Study Group \\ ${ }^{a}$ Department of Veterans Affairs Medical Center and b ${ }^{\mathrm{b}}$ Georgetown University School of Medicine, Washington, \\ D.C., ' Department of Veterans Affairs Medical Center, George Washington University, Washington, D.C., 'Wake \\ Forest School of Medicine, Winston-Salem, N.C., 'WFUHS Geriatric/Gerontology, Winston-Salem, Ohio, ${ }^{\mathrm{f}}$ University \\ of North Carolina, Chapel Hill, N.C., and ${ }^{9}$ Department of Veterans Affairs Medical Center, VA Clinical Center Network, \\ Memphis, Tenn., USA; ${ }^{h}$ McMaster Medical Center, Hamilton, Ont., Canada
}

\section{Key Words}

Type 2 diabetes mellitus · Chronic kidney disease · Stroke · Hypertension · Intensive antihypertensive therapy

\footnotetext{
Abstract

Background: Persons with chronic kidney disease (CKD) represent a population prone to cardiovascular disease (CVD) but vulnerable to adverse medication effects. We assessed the impact of intensive antihypertensive therapy on the cerebrovascular and other CVD outcomes in high-risk patients with type 2 diabetes and baseline CKD. Methods: Using current guideline criteria, 1,726 (36.9\%) of 4,678 participants in the Action to Control Cardiovascular Risk in Diabetes (ACCORD) blood pressure (BP) arm had mild to moderate CKD (CKD1-3B) at baseline. Participants of this study were randomized to intensive (systolic $<120 \mathrm{~mm} \mathrm{Hg}$ ) or standard (systolic $<140 \mathrm{~mm} \mathrm{Hg}$ ) BP goals. Fatal and non-fatal stroke were pre-specified secondary outcomes of the ACCORD
}

study. Results: Total cerebrovascular events were significantly higher in participants with baseline CKD (0.66\%/year) compared with participants free of CKD (0.28\%/year). A significantly higher rate of events was observed in CKD participants. Intensive antihypertensive therapy in participants without CKD at baseline resulted in a 55\% significant reduction of any stroke (hazard ratio $0.447 ; 95 \% \mathrm{Cl} 0.227-0.880$ ) and a $50 \%$ reduction of non-fatal stroke (hazard ratio 0.498 ; 95\% Cl 0.250-0.993). In participants with CKD at baseline, the occurrence of any stroke was reduced by $38 \%$ (hazard ratio $0.623 ; 95 \% \mathrm{Cl} 0.361-1.074$ ) and non-fatal stroke by $36 \%$ (hazard ratio $0.642 ; 95 \% \mathrm{Cl} 0.361-1.142)$. Test for interaction was NS between the 2 groups. Changes in other CVD outcomes did not reach statistical significance. Conclusions: These findings suggest that intensive antihypertensive therapy offers significant cerebrovascular protection in diabetic participants without CKD at baseline, but significant benefit to patients with CKD cannot be excluded.

(C) 2016 S. Karger AG, Base

\section{KARGER}

E-Mail karger@karger.com

www.karger.com/ajn
C 2016 S. Karger AG, Basel

$0250-8095 / 16 / 0434-0271 \$ 39.50 / 0$
Vasilios Papademetriou, MD

Department of Veterans Affairs Medical Center Georgetown University

50 Irving Street, NW, Washington, DC 20422 (USA)

E-Mail vpapademetriou@va.gov 


\section{Introduction}

Chronic kidney disease (CKD) affects approximately $11 \%$ of the US adult population and it is a marker of increased cardiovascular risk regardless of cause. The prevalence of earlier stages of CKD is more than 100 times greater than the prevalence of end-stage renal disease (ESRD) [1]. Recent data indicate that CKD may carry a cardiovascular disease (CVD) risk equivalent to diabetes and/or pre-existing CVD [2]. Prevention of CKD or retardation of disease progression has been advocated as another strategy toward cardiovascular protection [3]. Observational studies suggest a strong association between elevated blood pressure (BP), CVD and risk of renal function decline or ESRD, whereas individuals with $\mathrm{BP}$ below conventional thresholds show better preservation of renal function [4-6].

In the Systolic Blood Pressure Intervention Trial (SPRINT), CVD events and mortality were reduced and the results were similar across all major subgroups, including the $28 \%$ with CKD [7]. However, SPRINT excluded participants with known diabetes mellitus (DM). Therefore, the optimal BP goal for diabetic participants with CKD and more importantly with mild to moderate $\mathrm{CKD}$ has not been determined in prospective randomized trials. In the Action to Control Cardiovascular Risk in Diabetes (ACCORD) trial, participants with type II $\mathrm{DM}$ and hypertension had a significantly lower incidence of stroke with intensive BP therapy, but no significant reduction in other cardiovascular outcomes [8]. ACCORD enrolled participants with mild to moderate CKD (CKD I-III).

We therefore investigated the impact of CKD and intensive antihypertensive therapy on health outcomes, including cerebrovascular events in this high-risk population with type 2 diabetes.

\section{Methods}

ACCORD was a large (10,251 participants), multi-center, prospective, randomized study that aimed to evaluate the effects of intensive glycemic, lipid, and BP control vs. standard therapy in high-risk participants with type $2 \mathrm{DM}$. The study design included 3 intervention arms (glycemia, lipid, and BP) with all participants participating in the glycemia intervention [9]. Study participants were further randomized to the lipid and the BP studies in a double $2 \times 2$ factorial design $[8,10]$. The study design and the inclusion/ exclusion criteria have been reported in detail previously [8-10]. The BP trial included 4,733 participants with DM and treated or untreated systolic BP $130-180 \mathrm{~mm} \mathrm{Hg}$, and serum creatinine $\leq 1.5$ $\mathrm{mg} / \mathrm{dl}$, who were randomly assigned to standard antihypertensive therapy (target systolic $\mathrm{BP}<140 \mathrm{~mm} \mathrm{Hg}$ ) or intensive antihypertensive therapy (target systolic BP $<120 \mathrm{~mm} \mathrm{Hg}$ ).

The study was performed in accordance with the Helsinki Declaration, and protocol approval was granted by the IRB or Ethics Committees of all participating center, and by a review panel at the NHLBI. All participants were informed in detail and then they consented to participate in the study.

Estimated glomerular filtration rate (eGFR) was calculated by using the Modification of Diet in Renal Disease (MDRD) formula. A 5-stage classification system was used to define CKD categories according to KDIGO recommendations [11]. Stage I CKD was defined as normal eGFR $\left(\geq 90 \mathrm{ml} / \mathrm{min} / 1.73 \mathrm{~m}^{2}\right)$ and increased albumin excretion (urine albumin/creatinine ratio $\geq 30 \mu \mathrm{g} / \mathrm{mg}$ ). Stage II CKD was defined as eGFR between 60 and $89 \mathrm{ml} / \mathrm{min} / 1.73 \mathrm{~m}^{2}$ and urine albumin/creatinine ratio $\geq 30 \mu \mathrm{g} / \mathrm{mg}$. Stage III was defined as eGFR between 30 and $59 \mathrm{ml} / \mathrm{min} / 1.73 \mathrm{~m}^{2}$ with or without albuminuria. Stage III CKD was further subdivided into CKD IIIA (eGFR 30-44) and CKD IIIB (eGFR 45-59). Study participants with stages I and II CKD were considered participants with mild CKD, while participants with stage III CKD were considered participants with moderate CKD. Most CKD III were CKD IIIB patients participants with stage IV and V CKD were not included in the ACCORD trial.

A composite end-point including both non-fatal and fatal CVD events (first occurrence of non-fatal myocardial infarction (MI), non-fatal stroke, and cardiovascular death) was used as the primary end point of the study. Pre-specified secondary outcomes included cardiovascular and total mortality, fatal and non-fatal stroke, non-fatal MI, non-fatal and fatal congestive heart failure, major coronary events, and revascularization [9].

\section{Statistical Analysis}

For tables 1 and 2, continuous variables are expressed as mean \pm SD. When the distribution was highly skewed, the median was used and interquartile range is reported. Categorical variables are reported as numbers and percentages. Baseline characteristics between CKD and non-CKD participants as well as intensive vs. standard therapy in each group were compared using chi square, 2-sample t tests, or Fisher's exact tests.

Cox proportional hazard models were used for the analyses of the primary and secondary outcomes in the intensive vs. standard $\mathrm{BP}$ control groups and according to whether $\mathrm{CKD}$ was present or not. Occurrences of these outcomes were compared with the use of hazard ratios and 95\% CIs. Two-sided p values were obtained from likelihood ratio tests from Cox proportional-hazards regression analyses. Event rates are expressed as the percentage of events per 100 follow-up years, taking into account censoring of followup data.

Cox models contained a term representing study group assignments plus terms accounting for the following prespecified stratification variables: assignment to the lipid trial, assignment to the intensive glycemic intervention, assignment to receive fibrate in the lipid trial, the 7 clinical-center networks, the presence or absence of a previous cardiovascular event, and the following baseline covariates: cardiovascular history, clinical center network, age (years), female (yes/no), BMI $\left(\mathrm{kg} / \mathrm{m}^{2}\right), \mathrm{HbAlc}(\%)$, systolic BP, smoking status (yes/no), insulin use (yes/no), and antihypertensive use (yes/no).

Subgroup analyses for the consistency of the effects among subgroups of study participants were performed using interaction tests between subgroups and therapy effects. 
Table 1. Baseline characteristics of entire BP cohort and by baseline CKD status

\begin{tabular}{|c|c|c|c|c|}
\hline \multirow[t]{2}{*}{ Overall $(\mathrm{n}=4,678)$} & & \multicolumn{2}{|l|}{ Baseline CKD status } & \multirow[t]{2}{*}{$\mathrm{p}$ value } \\
\hline & & $\begin{array}{l}\text { no CKD } \\
(\mathrm{n}=2,952)\end{array}$ & $\begin{array}{l}\text { with CKD } \\
(\mathrm{n}=1,726)\end{array}$ & \\
\hline Age, years & $62.2 \pm 6.9$ & $61.6 \pm 6.5$ & $63.2 \pm 7.3$ & $<0.0001$ \\
\hline Female sex & $2,231(47.7)$ & $1,455(49.3)$ & $776(45.0)$ & 0.0042 \\
\hline Race/ethnicity & & & & 0.5512 \\
\hline African American & $1,110(23.7)$ & $695(23.5)$ & $415(24.0)$ & \\
\hline White & $2,757(58.9)$ & $1,752(59.3)$ & $1,005(58.2)$ & \\
\hline Hispanic & $320(6.8)$ & $191(6.5)$ & $129(7.5)$ & \\
\hline Other & $491(10.5)$ & $314(10.6)$ & $177(10.3)$ & \\
\hline Weight, kg & $92.0 \pm 18.5$ & $91.8 \pm 18.4$ & $92.4 \pm 18.7$ & 0.2621 \\
\hline BMI, $\mathrm{kg} / \mathrm{m}^{2}$ & $32.2 \pm 5.6$ & $32.0 \pm 5.5$ & $32.3 \pm 5.7$ & 0.0739 \\
\hline Smoking status & & & & $<0.0001$ \\
\hline Never & $2,093(44.8)$ & $1,369(46.4)$ & $724(42.0)$ & \\
\hline Former & $1,959(41.9)$ & $1,234(41.8)$ & $725(42.1)$ & \\
\hline Current & $621(13.3)$ & $347(11.8)$ & $274(15.9)$ & \\
\hline Serum creatinine, mg/dl & $0.9 \pm 0.2$ & $0.8 \pm 0.2$ & $1.0 \pm 0.3$ & $<0.0001$ \\
\hline CKD & & & & $<0.0001$ \\
\hline No CKD & $2,952(63.1)$ & $2,952(100)$ & $0(0.0)$ & \\
\hline Stage I & $693(14.8)$ & $0(0.0)$ & $693(40.2)$ & \\
\hline Stage II & $632(13.5)$ & $0(0.0)$ & $632(36.6)$ & \\
\hline Stage III & $401(8.6)$ & $0(0.0)$ & $401(23.2)$ & \\
\hline Serum glucose, mg/dl & $174.6 \pm 57.6$ & $171.3 \pm 52.6$ & $180.1 \pm 65.0$ & $<0.0001$ \\
\hline HbAlc, \% & $8.3 \pm 1.1$ & $8.3 \pm 1.0$ & $8.5 \pm 1.1$ & $<0.0001$ \\
\hline Duration of DM, years & $11.0 \pm 8.0$ & $10.2 \pm 7.6$ & $12.5 \pm 8.4$ & $<0.0001$ \\
\hline Systolic BP & $139.2 \pm 15.8$ & $137.2 \pm 15.0$ & $142.5 \pm 16.7$ & $<0.0001$ \\
\hline Diastolic BP & $75.9 \pm 10.4$ & $76.2 \pm 10.1$ & $75.5 \pm 10.9$ & 0.0251 \\
\hline Heart rate & $73.1 \pm 11.6$ & $72.8 \pm 11.3$ & $73.6 \pm 12.1$ & 0.0175 \\
\hline History of CVD & $1,577(33.7)$ & $897(30.4)$ & $680(39.4)$ & $<0.0001$ \\
\hline History of $\mathrm{CHF}$ & $201(4.3)$ & $95(3.3)$ & $106(6.2)$ & $<0.0001$ \\
\hline Insulin & $1,728(36.9)$ & $979(33.2)$ & $749(43.4)$ & $<0.0001$ \\
\hline Merformin & $2,682(57.3)$ & $1,724(58.4)$ & $958(55.5)$ & 0.0532 \\
\hline Any sulfonylurea & $2,220(47.5)$ & $1,435(48.6)$ & $785(45.5)$ & 0.0386 \\
\hline Any antihypertensive agent & $4,088(87.4)$ & $2,507(84.9)$ & $1,581(91.6)$ & $<0.0001$ \\
\hline ACE-inhibitors & $2,439(52.1)$ & $1,503(50.9)$ & $936(54.2)$ & 0.0285 \\
\hline ARBs & $791(16.9)$ & $482(16.3)$ & $309(17.9)$ & 0.1656 \\
\hline Any thiazide diuretic & $1,000(21.4)$ & $623(21.1)$ & $377(21.8)$ & 0.5524 \\
\hline Beta blockers & $1,196(25.6)$ & $674(22.8)$ & $522(30.2)$ & $<0.0001$ \\
\hline \multicolumn{5}{|l|}{ CCBs } \\
\hline Dihydropyridine & $517(11.1)$ & $274(9.3)$ & $243(14.1)$ & $<0.0001$ \\
\hline Non-dihydropyridine & $347(7.4)$ & $178(6.0)$ & $169(9.8)$ & $<0.0001$ \\
\hline Aspirin & $2,447(52.3)$ & $1,535(52.0)$ & $912(52.8)$ & 0.5787 \\
\hline Statin & $3,035(64.9)$ & $1,909(64.7)$ & $1,126(65.2)$ & 0.6938 \\
\hline Fibrate & $307(6.6)$ & $181(6.1)$ & $126(7.3)$ & 0.1193 \\
\hline Potassium & $4.5 \pm 0.7$ & $4.4 \pm 0.4$ & $4.5 \pm 1.0$ & $<0.0001$ \\
\hline LDL & $110.0 \pm 36.7$ & $110.2 \pm 35.9$ & $109.7 \pm 38.1$ & 0.6214 \\
\hline \multicolumn{5}{|l|}{ HDL } \\
\hline Female & $51.3 \pm 13.8$ & $52.0 \pm 13.7$ & $50.0 \pm 13.9$ & 0.0016 \\
\hline Male & $41.7 \pm 11.7$ & $42.0 \pm 11.2$ & $41.3 \pm 12.5$ & 0.1697 \\
\hline Triglycerides & $147.0(98.0-226.0)$ & $143.0(95.0-212.0)$ & $158.0(103.0-249.0)$ & $<0.0001$ \\
\hline
\end{tabular}


Table 2. Baseline characteristics of study participants by baseline CKD status and treatment arm

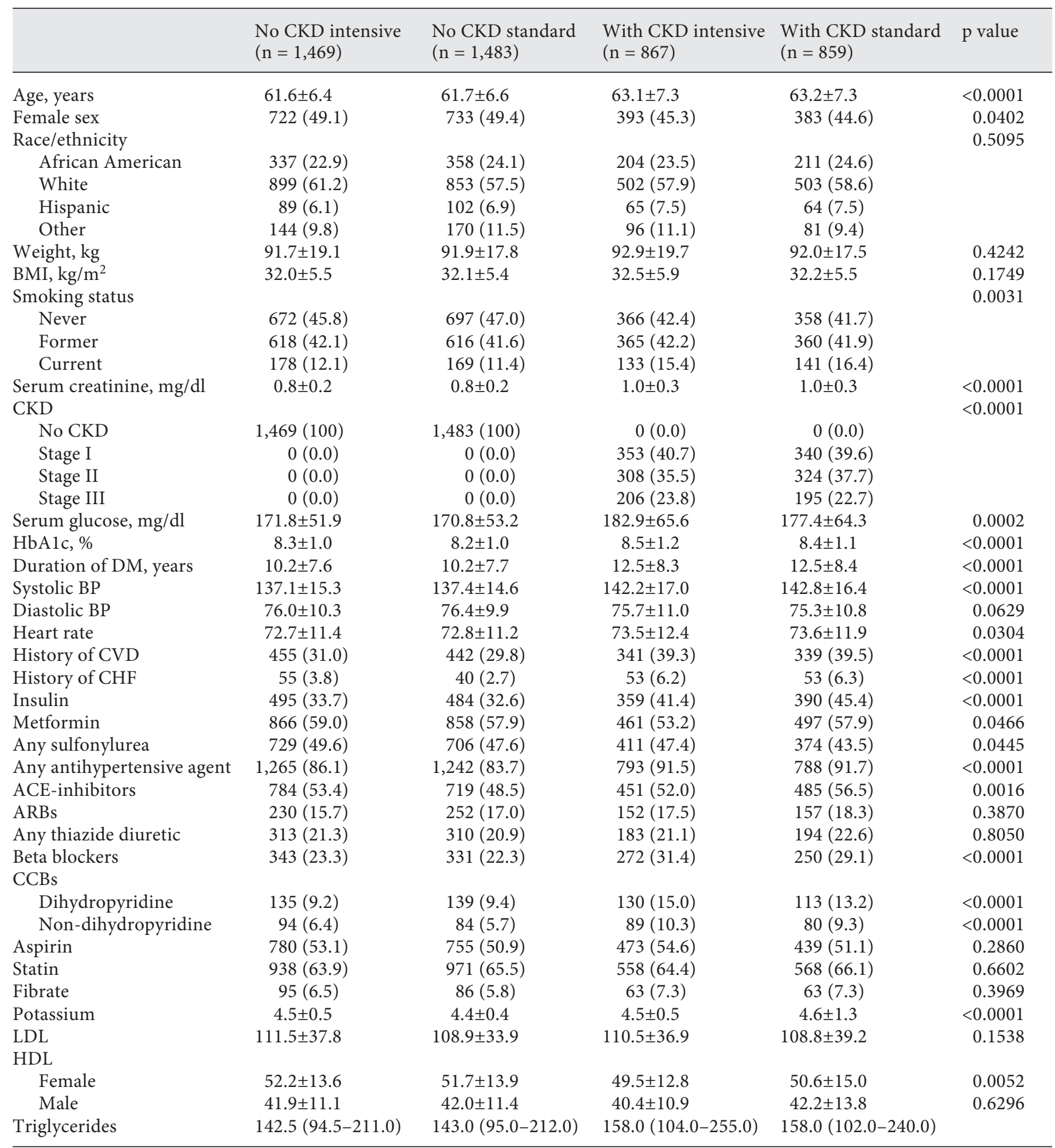


Fig. 1. Systolic BP according to BP arm and CKD status at baseline.

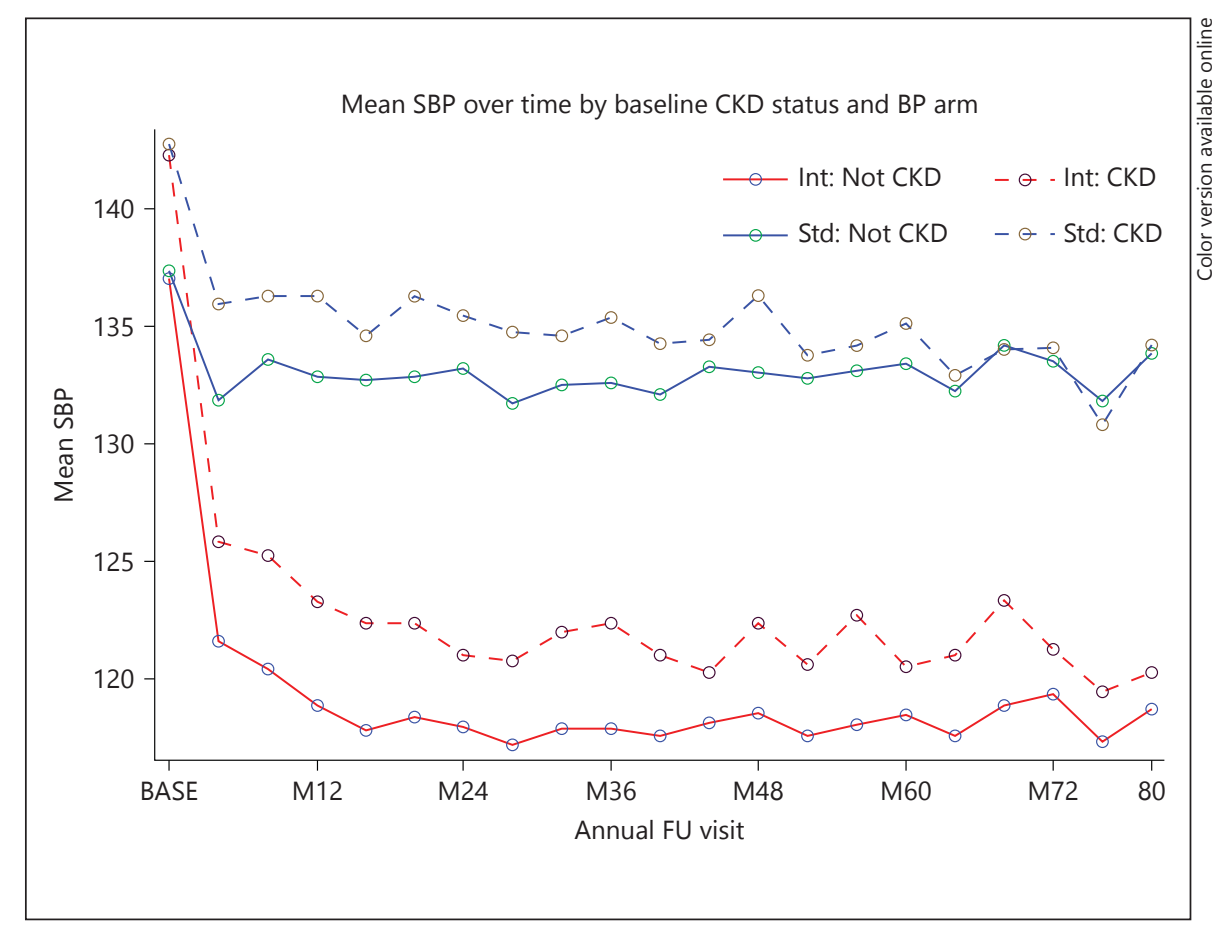

No adjustments were made for multiple testing. Nominal $\mathrm{p}$ values are reported throughout as simple guides to possible associations. All analyses were performed at a central coordinating center using SAS 9.3 software (SAS Institute, Cary, N.C., USA).

\section{Results}

\section{Baseline}

Data recording renal function were available for 4,678 of the 4,733 participants in the BP arm of the ACCORD study. More than $60 \%$ of them were free of CKD $(2,952$ participants; $63.1 \%)$, while the remaining 1,726 participants $(36.9 \%)$ had some degree of CKD. More specifically, 693 participants $(14.8 \%)$ were classified as Stage I CKD, 632 participants (13.5\%) as stage II CKD and 401 $(8.6 \%)$ as stage III CKD.

The key baseline characteristics of study participants according to CKD status at baseline are described in table 1. Participants with CKD were older by about 1.5 years on average, had higher waist circumference, were more frequently current smokers, had greater duration of diabetes and higher HbA1c levels, higher systolic BP values and higher rates for history of heart failure and CVD. For the treatment of diabetes, participants with CKD used insulin and sulfonylureas more frequently and metformin less frequently than participants without CKD baseline. Antihypertensive agents were used more frequently by participants with CKD.

Table 2 presents the main baseline characteristics according to BP arm (intensive vs. standard) and CKD status at baseline. Differences in baseline parameters between CKD and non-CKD participants are observed in each BP arm, similar to the total population. Importantly, however, no significant differences between the $2 \mathrm{BP}$ arms (intensive vs. standard therapy) were observed, both for CKD and for non-CKD participants.

\section{Blood Pressure}

At baseline, systolic BP was significantly higher in the CKD group as compared to the non-CKD group (142.5 \pm 16.7 vs. $137.2 \pm 15.0 \mathrm{~mm} \mathrm{Hg}, \mathrm{p}<0.0001$; table 1) but very similar between intensive and standard arms in both groups (table 2). BP was significantly reduced in the intensive arm in both CKD and non-CKD participants (fig. 1). At the end of the study, systolic BP in the standard arm was similar between CKD and non-CKD participants $(134 \mathrm{~mm} \mathrm{Hg})$ and much lower in the intensive arm (122 mm Hg in CKD, $119 \mathrm{~mm} \mathrm{Hg}$ in non-CKD participants). Of note, BP levels were consistently lower throughout the study in non-CKD as compared to CKD participants both in the intensive and standard treatment arms (fig. 1). 


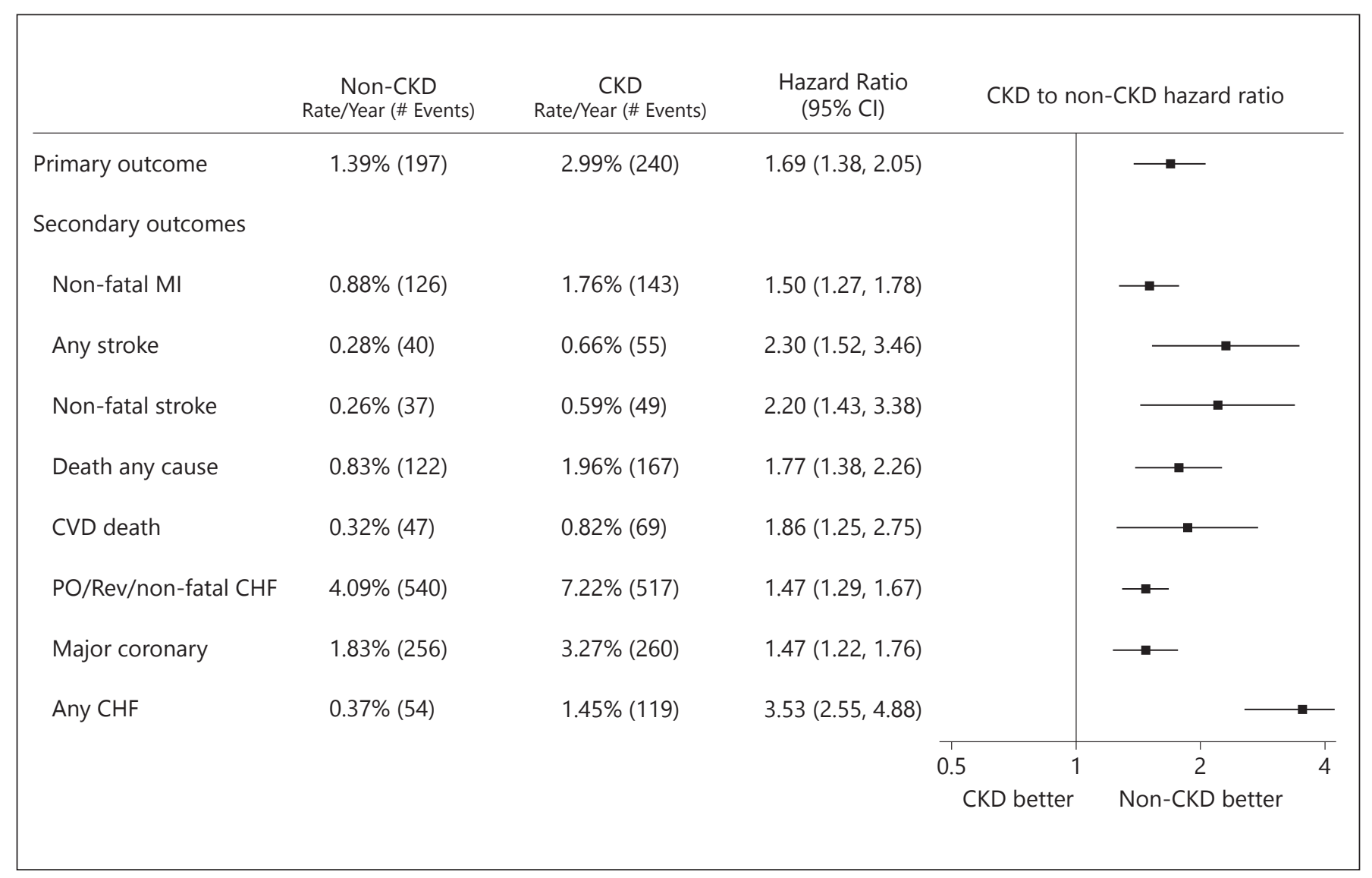

Fig. 2. Rates of primary and secondary outcomes according to CKD status at baseline among participants in the $\mathrm{BP}$ arm of the ACCORD study.

\section{Outcomes}

Figure 2 presents the rates for the primary and secondary CVD outcomes according to CKD status at baseline. All outcomes occurred at a much higher annual rate in CKD participants as compared to non-CKD participants. The primary end point occurred at a rate of $2.99 \% / y e a r$ in the CKD vs. $1.39 \%$ /year in the non-CKD participants ( $\mathrm{p}<$ $0.0001)$. Similar differences were noted in all secondary outcomes that include non-fatal MI, any and non-fatal stroke, CV and all-cause mortality, major coronary events and fatal and non-fatal heart failure (fig. 2). Importantly, the risk of any stroke was more than 2.3 times higher in CKD as compared to non-CKD participants (hazard ratio 2.3; 95\% CI 1.5-3.5; p < 0.0001) and that of non-fatal stroke was more than 2.2 times higher in CKD as compared to non-CKD participants (hazard ratio 2.2; 95\% CI $1.4-3.4 ; \mathrm{p}<0.0001)$.

The rates for the primary and secondary outcomes in participants without CKD according to assignment treatment group are presented in figure $3 \mathrm{a}$. In this group of participants, an intensive decrease in BP was associated with a significantly lower rate of any stroke (55\% lower) and in non-fatal stroke (50\% lower) when compared to the standard BP-lowering group (hazard ratio 0.447 and 95\% CI $0.227-0.880$ for any stroke; hazard ratio 0.498 and $95 \%$ CI $0.250-0.993$ for non-fatal stroke, respectively). A trend toward lower rates for the primary outcome, non-fatal MI and fatal or non-fatal heart failure was observed with intensive therapy compared to standard therapy, but differences did not reach statistical significance.

In participants with CKD at baseline (fig. 3b), an intense reduction in $\mathrm{BP}$ was associated with a trend toward reduced rates for the primary and all secondary outcomes, without reaching statistical significance. The reduction in outcomes was larger for any stroke (38\%) and non-fatal stroke (36\%), but statistical significance was not reached (hazard ratio 0.623 and 95\% CI $0.361-1.074$ for any stroke; hazard ratio 0.642 and 95\% CI 0.361-1.142 for non-fatal stroke, respectively). 


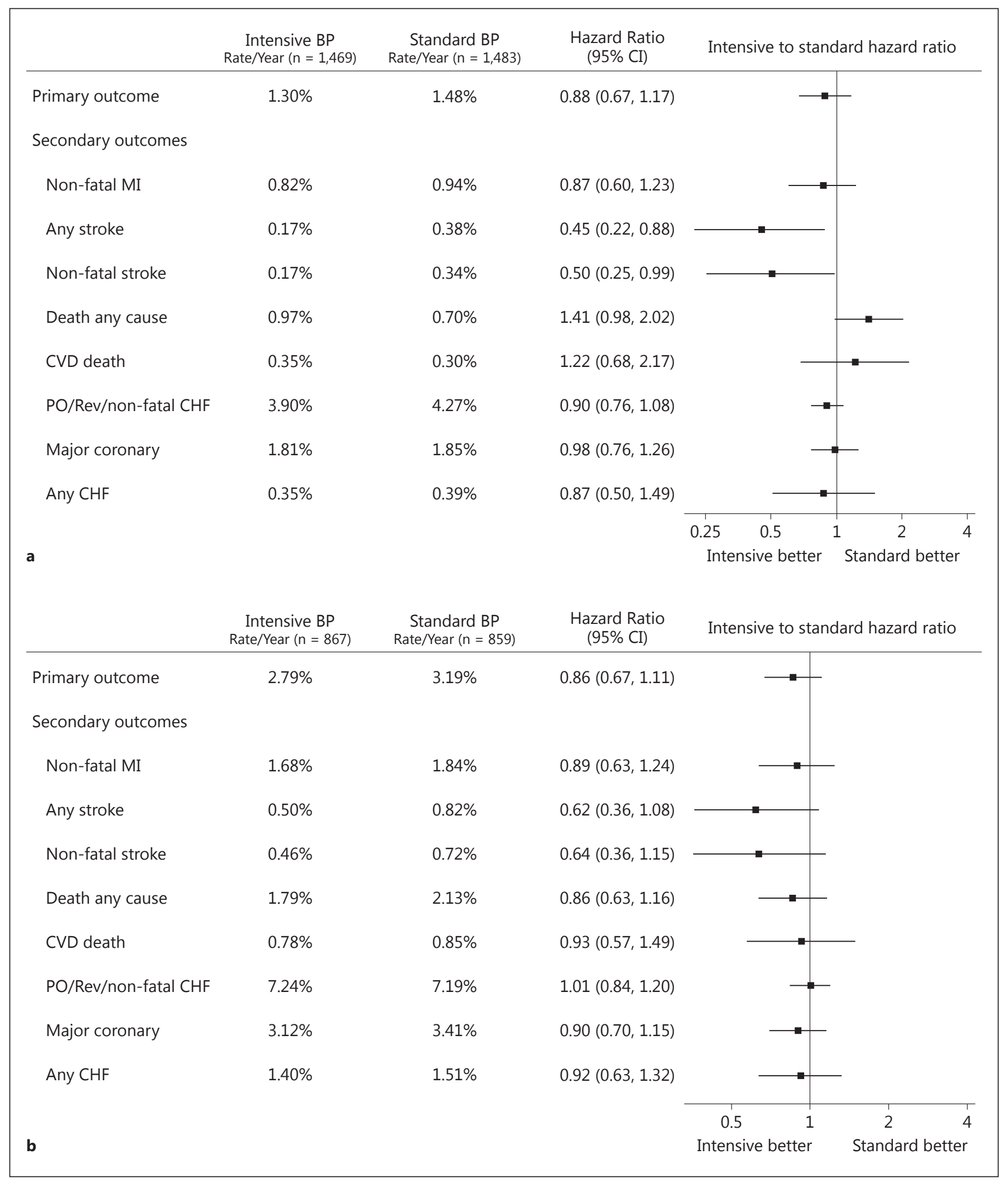

Fig. 3. Rates for the primary and secondary outcomes by treatment group (intensive vs. standard) in participants without CKD (a) and with CKD (b) at baseline. 
Table 3. Serious adverse events categories by BP arm and CKD status at baseline

\begin{tabular}{lcccc}
\hline AE_Category & int_No CKD_pct & std_No CKD_pct & int_With CKD_pct & std_With CKD_pct \\
\hline Cardiovascular & $26(1.77)$ & $7(0.47)$ & $29(3.34)$ & $14(1.63)$ \\
Digestive & $0(0.0)$ & $2(0.13)$ & $0(0.0)$ & $2(0.23)$ \\
Endocrine & $1(0.07)$ & $2(0.13)$ & $0(0.0)$ & $1(0.12)$ \\
Excretory & $2(0.14)$ & $2(0.13)$ & $10(1.15)$ & $1(0.12)$ \\
Immune/lymphatic & $3(0.20)$ & $2(0.13)$ & $5(0.58)$ & $4(0.47)$ \\
Nervous/sensory & $3(0.20)$ & $0(0.0)$ & $1(0.12)$ & $2(0.23)$ \\
Respiratory & $0(0.0)$ & $0(0.0)$ & $2(0.23)$ & $3(0.35)$ \\
Skeletal & $0(0.0)$ & $1(0.07)$ & $0(0.0)$ & $1(0.12)$ \\
Other & $5(0.34)$ & $1(0.07)$ & $6(0.69)$ & $6(0.70)$ \\
None & $1,429(97.3)$ & $1,466(98.9)$ & $814(93.9)$ & $825(96.0)$ \\
\hline
\end{tabular}

The number of serious adverse events was significantly higher in CKD compared with non-CKD participants ( 87 vs. 57 , respectively) and in intensively treated participants (93 vs. 51 for intensive vs. standard therapy, respectively). The organ-specific serious adverse events that occurred during the study according to CKD status at baseline and $\mathrm{BP}$ arm are depicted in table 3.

\section{Discussion}

Results of these subgroup analyses of the ACCORD data indicate 2 major findings. First, diabetic participants with hypertension and mild/moderate CKD have significantly more $\mathrm{CV}$ events as compared to similar participants with no CKD. Second, intensive BP control in participants with no CKD results in substantial and significant reduction of cerebrovascular events, while the same level of BP control in persons with baseline CKD shows a nonsignificant similar trend for reduction of these events. These findings are important and may have clinical implications.

First, the increased risk for cardiovascular events in participants with CKD was true for the primary end point, fatal and non-fatal MI, cardiovascular and allcause mortality, any and non-fatal stroke and heart failure. This finding is in line with prior data indicating increased cardiovascular risk in participants with CKD and other comorbidities [1, 12-14]. The risk in general and mixed populations is proportional to severity of CKD and increases more than 18 -fold in participants with ESRD $[13,15]$. Data however, for diabetic participants with hypertension and mild CKD are scarce and it is not known if indeed they fall in the same risk category as that of other subgroups with diabetic participants. Data from this study strongly suggest that even mild CKD is a strong risk factor for cardiovascular events in these participants with diabetes and hypertension. Diabetic participants with no evidence of CKD do not carry as much risk.

The second and likely more important finding of our study is the effect of intensive BP control on cardiovascular events in diabetic participants, with and without mildto-moderate $\mathrm{CKD}$. In both groups, tight $\mathrm{BP}$ control showed trends for improvement in cardiovascular events, albeit non-statistically significant for most of the outcomes. The only significant finding was a 55\% reduction of total and $50 \%$ of non-fatal stroke in the non-CKD group. In the diabetic-hypertensive CKD population, intensive antihypertensive therapy did show a $30 \%$ risk reduction in total and non-fatal stroke and a $20 \%$ improvement in all-cause mortality but neither reached statistical significance.

The fact, however, that trends for outcome changes were in the same direction and numerically similar between the 2 groups indicates that intensive BP control may have similar impacts in participants with and without CKD (test of interaction = NS), but most likely both subgroups were underpowered to show significant reductions in all health outcomes in this ACCORD population. Larger studies with longer follow-up will be needed to accurately test this hypothesis. We could find very limited data on the impact of intensive lowering of BP in participants with both diabetes and CKD. Future analyses from the SPRINT study will shed more light into the question whether intensive BP control benefits hypertensive participants with baseline high-risk CKD. Although the SPRINT study had similar design with ACCORD, diabetic participants were not included [7].

The findings of this study showing that intensive reduction in BP reduces cerebrovascular events but has lim- 
ited impact on CHD events are consistent with findings from other randomized controlled clinical trials of patients with hypertension and diabetes.

In the factorial design ADVANCE trial, 11,140 type 2 diabetics were randomized to fixed combination of 2 antihypertensive medications or matching placebo. In the treated group average, BP was reduced to 135/75 and in the placebo group to $140 / 77 \mathrm{~mm} \mathrm{Hg}$. Results showed a significant $9 \%$ overall reduction of major macrovascular and microvascular events [16]. There was also significant relative risk reduction in the total number of coronary events (14\%) and an 18\% reduction in cardiovascular death. There was a minor nonsignificant $6 \%$ reduction in stroke. In the Systolic Hypertension in the Elderly Program, in the subgroup of 583 patients with diabetes, BP in the treatment group was $146 / 70$ $\mathrm{mm} \mathrm{Hg}$ as compared to 155/74 in the placebo group. All major CVD events were reduced by $34 \%$ [17], whereas stroke was reduced by $26 \%$. This was a similar relative risk reduction as in participants without diabetes, but the absolute risk reduction was twice as great for diabetic participants. In the Hypertension Optimal Treatment trial, over 18,000 participants were randomized to a diastolic BP goal of $<90,<85$ or $<80 \mathrm{~mm} \mathrm{Hg}$ [18]. Although the overall study was negative, results in the diabetic subgroup $(\mathrm{n}=1,501)$ were positive. All major CVD events were reduced significantly by $51 \%$ ( $\mathrm{p}=$ 0.005 ) in those randomized to diastolic BP goal of $\leq 80 \mathrm{~mm} \mathrm{Hg}$ compared to a goal of $\leq 90 \mathrm{~mm} \mathrm{Hg} ; 12$ vs. 24 events/1,000 patient-years. In the United Kingdom Prospective Diabetes Study, 1,148 participants with hypertension and type 2 diabetes were randomized to either tighter BP control $<150 / 85 \mathrm{~mm} \mathrm{Hg}$ or less tight BP control $<180 / 105 \mathrm{~mm} \mathrm{Hg}$ [19]. After 9 years of follow-up, the average BP was reduced to $144 / 82$ vs. $154 / 87$ $\mathrm{mm} \mathrm{Hg}$. Diabetes-related end points were reduced by $24 \%(\mathrm{p}=0.005)$, death related to diabetes by $32 \%(\mathrm{p}=$ $0.019)$, strokes by $44 \%(\mathrm{p}=0.013)$, and microvascular end points by $37 \%(\mathrm{p}=0.009)$ in the tighter control group. All-cause mortality was lower by $18 \%$ and MI by $21 \%$ in the more intensively treated group, though not statistically significant. In the Systolic Hypertension in Europe trial, in 492 participants with diabetes, the mean systolic BP was reduced to $153 / 77$ vs. $162 / 82 \mathrm{~mm} \mathrm{Hg}$ with significant reductions in CVD mortality, all CVD events and stroke [20].

In a recent systematic review/meta-analysis [21] that included 100,354 patients with hypertension and type $2 \mathrm{DM}$ from 40 studies, it was found that for each $10 \mathrm{~mm}$ $\mathrm{Hg}$ lower systolic BP there was a significant $13 \%$ reduc-

Intensive BP Control in Patients with DM and $\mathrm{CKD}$ tion in all-cause mortality, $11 \%$ reduction in cardiovascular events, $12 \%$ reduction in coronary heart disease events and $27 \%$ reduction in the incidence of stroke. Benefit in all cardiovascular events was realized in all subgroups with baseline BP $>140 \mathrm{~mm} \mathrm{Hg}$ systolic with treated pressure reduced to about $135 \mathrm{~mm} \mathrm{Hg}$, except for stroke. Significant reduction in stroke was found for patients with baseline BP $>140$ systolic and $<140 \mathrm{~mm} \mathrm{Hg}$ systolic. In fact, the former group achieved an after-treatment systolic BP of $138 \mathrm{~mm} \mathrm{Hg}$ and a reduction of stroke by $24 \%$, and the latter group achieved a systolic BP of 121 $\mathrm{mm} \mathrm{Hg}$ and a reduction in stroke by $28 \%$. These data indicate that there is benefit for stroke reduction with achieved systolic BP around $120 \mathrm{~mm} \mathrm{Hg}$, which is very similar with the findings of our study. Unfortunately, data in this meta-analysis did not stratify patients by CKD status.

To conclude, in most studies aforementioned, achieved $\mathrm{BP}$ with intensive treatment was around $135 / 75 \mathrm{~mm} \mathrm{Hg}$. Yet treatment showed substantial risk reduction in cardiovascular events and stroke in a few studies that achieved average systolic BP of $121 \mathrm{~mm} \mathrm{Hg}$. In our study, the intensive $\mathrm{BP}$ arm achieved mean reduction to $119 \mathrm{~mm} \mathrm{Hg}$ and showed significant improvement in stroke conditions. This is also consistent with the results of recent SPRINT trial in which intensive therapy led to $30 \%$ relative risk reduction in fatal and non-fatal major cardiovascular outcomes and death from any cause in high risk participants without diabetes [7].

In the CKD population, data from randomized controlled trials on intensity of antihypertensive therapy on cardiovascular outcomes are scarce. The MDRD and AASK trials focused on progression of CKD as primary outcomes in non-diabetic CKD participants [22, 23]. Neither trial found any significant difference comparing mean GFR from baseline to end of treatment between standard $(140 / 90 \mathrm{~mm} \mathrm{Hg})$ and intensive $(125 / 75 \mathrm{~mm} \mathrm{Hg})$ BP targets. It should be noted, however, that neither study had sufficient statistical power to evaluate the effects of more intensive BP intervention on major cardiovascular outcomes.

In our analysis, intensive BP control showed a significant risk reduction in stroke in the non-CKD group and a similar but nonsignificant trend in the CKD group. Lack of statistical significance could be due to a small number of participants. Thus, the trends in outcome change were concordant in both groups, which is at variance with the results of the previous analysis we did on the glycemic arm of ACCORD. In that analysis we found that intensive glycemic control in patients with mild-to-moderate CKD 
resulted in marked increase in $\mathrm{CV}$ and all-cause mortality [23]. The number of serious adverse events noted in this study was significantly higher in CKD compared with non-CKD participants and in intensively treated patients compared to standard treated participants as observed in previous trials, in the main ACCORD BP trial [8], and in a prior analysis of $\mathrm{ACCORD}$ according to $\mathrm{CKD}$ status [24].

In summary, this study demonstrates that in patients with hypertension and type $2 \mathrm{DM}$, even mild CKD is associated with marked increase in $\mathrm{CV}$ events. Intensive $\mathrm{BP}$ treatments in patients with no CKD results in marked reduction of cerebrovascular events and outcomes in patients with CKD may not be different.

\section{Acknowledgment}

The authors like to acknowledge the help of Paola Chrysostomou who prepared this manuscript.

\section{Source of Funding}

NHLBI (ClinicalTrials.gov number, NCT00000620).

\section{Disclosure Statement}

Dr. Cushman has received institutional grant support from Eli Lilly and Boehringer-Ingelheim, and is an unpaid consultant to Takeda.

\section{References}

1 Levey AS, Coresh J, Balk E, et al: National kidney foundation practice guidelines for chronic kidney disease: evaluation, classification, and stratification. Ann Intern Med 2003;139: 137-147.

2 Tonelli M, Muntner P, Lloyd A, et al: Risk of coronary events in people with chronic kidney disease compared with those with diabetes: a population-level cohort study. Lancet 2012;380:807-814.

3 Couser WG, Riella MC: World kidney day 2011: protect your kidneys, save your heart. Kidney Int 2011;79:483-485.

4 Kidney Disease Outcomes Quality Initiative (K/DOQI): K/DOQI clinical practice guidelines on hypertension and antihypertensive agents in chronic kidney disease. Am J Kidney Dis 2004;43(5 suppl 1):S1-S290.

5 Sarafidis PA, Barkis GL: Kidney disease and hypertension; in Lip GY, Hall JE (eds): Comprehensive Hypertension. Philadelphia, Mosby Elsevier, 2007, pp 607-619.

6 National Kidney Foundation: KDIGO clinical practice guideline for management of blood pressure in chronic kidney disease. Kidney Int Suppl 2013;2:337-414.

7 SPRINT Research Group, Wright JT Jr, Williamson JD, Whelton PK, et al: A randomized trial of intensive versus standard blood-pressure control. N Engl J Med 2015;373:21032116.

8 ACCORD Study Group, Cushman WC, Evans GW, Byington RP, et al: Effects of intensive blood-pressure control in type 2 diabetes mellitus. N Engl J Med 2010;362:1575-1585.

9 Action to Control Cardiovascular Risk in Diabetes Study Group, Gerstein HC, Miller ME, Byington RP, et al: Effects of intensive glucose lowering in type 2 diabetes. $\mathrm{N}$ Engl J Med 2008;358:2545-2559.
10 ACCORD Study Group, Ginsberg HN, Elam $\mathrm{MB}$, Lovato LC, et al: Effects of combination lipid therapy in type 2 diabetes mellitus. N Engl J Med 2010;362:1563-1574.

11 KDIGO: KDIGO 2012 clinical practice guideline for the evaluation and management of chronic kidney disease. Kidney Int 2013;3:S1S150.

12 Coresh J, Selvin E, Stevens LA, et al: Prevalence of chronic kidney disease in the United States. JAMA 2007;298:2038-2048.

13 Go AS, Chertow GM, Fan D, et al: Chronic kidney disease and the risks of death, cardiovascular events, and hospitalization. $\mathrm{N}$ Engl J Med 2004;351:1296-1305.

14 Chronic Kidney Disease Prognosis Consortium, Matsushita K, van der Velde M, Astor BC, Woodward M, Levey AS, et al: Association of estimated glomerular filtration rate and albuminuria with all-cause and cardiovascular mortality in general population cohorts: a collaborative meta-analysis. Lancet 2010;375:2073-2081.

15 Roderick PJ, Atkins RJ, Smeeth L, et al: CKD and mortality risk in older people: a community-based population study in the United Kingdom. Am J Kidney Dis 2009;53:950-960.

16 Patel A, MacMahon S, Chalmers J, et al: Effects of a fixed combination of perindopril and indapamide on macrovascular and microvascular outcomes in patients with type 2 diabetes mellitus (the ADVANCE trial): a randomised controlled trial. Lancet 2007;370:829-840.

17 Prevention of stroke by antihypertensive drug treatment in older persons with isolated systolic hypertension. Final results of the Systolic Hypertension in the Elderly Program (SHEP). SHEP Cooperative Research Group. JAMA 1991;265:3255-3264.
18 Hansson L, Zanchetti A, Carruthers SG, et al: Effects of intensive blood-pressure lowering and low-dose aspirin in patients with hypertension: principal results of the Hypertension Optimal Treatment (HOT) randomised trial. HOT Study Group. Lancet 1998;351:17551762.

19 Adler AI, Stratton IM, Neil HA, et al: Association of systolic blood pressure with macrovascular and microvascular complications of type 2 diabetes (UKPDS 36): prospective observational study. BMJ 2000;321:412-419.

20 Staessen JA, Fagard R, Thijs L, et al: Randomised double-blind comparison of placebo and active treatment for older patients with isolated systolic hypertension. The Systolic Hypertension in Europe (Syst-Eur) Trial Investigators. Lancet 1997;350:757-764.

21 Emdin CA, Rahimi K, Neal B, Callender T, Perkovic V, Patel A: Blood pressure lowering in type 2 diabetes: a systematic review and meta-analysis. JAMA 2015;313:603-615.

22 Klahr S, Levey AS, Beck GJ, et al: The effects of dietary protein restriction and blood-pressure control on the progression of chronic renal disease. Modification of Diet in Renal Disease Study Group. N Engl J Med 1994;330: 877-884.

23 Appel LJ, Wright JT Jr, Greene T, et al: Intensive blood-pressure control in hypertensive chronic kidney disease. N Engl J Med 2010; 363:918-929.

24 Papademetriou V, Lovato L, Doumas M, et al: Chronic kidney disease and intensive glycemic control increase cardiovascular risk in patients with type 2 diabetes. Kidney Int 2015; 87:649-659. 